\title{
A Medium Frequency Transformer Design Tool with Methodologies Adated to Various Structures
}

\author{
Alexis Fouineau \\ SuperGrid Institute \\ 69100 Villeurbanne, France \\ Email: alexis.fouineau@supergrid-institute.com \\ Martin Guillet \\ SuperGrid Institute \\ 69100 Villeurbanne, France \\ Email: martin.guillet@,supergrid-institute.com \\ Bruno Lefebvre \\ SuperGrid Institute \\ 69100 Villeurbanne, France \\ Email: bruno.lefebvre@supergrid-institute.com
}

\begin{abstract}
A complete and generic medium frequency transformer (MFT) design methodology is presented in this paper, which can be applied to many transformer structures. Models were found or developed to cover every necessary calculation, with a focus on the balance between computation time and accuracy, leading to a fast and efficient design tool. A lot of MFT designs are available at the end and the possibility to choose the best candidate is offered. A multi-megawatt offshore windfarm converter application has been chosen to show the design optimization procedure of the MFT inside such a converter. The best potential design found is then validated by numerous finite element simulations to validate the models used. This procedure is then repeated for various MFT structures in order to realize a quantitative comparison of many different technological ehoieeschoices' combinations. This study can give insights on the best technological choices to be used for MFT, and also shows significant differences on performances between structures.
\end{abstract}

Keywords-Power Transformers; modeling; design optimization.

\section{INTRODUCTION}

Solid-state transformers (SST) are a promising technology to replace and enhance conventional transformers or to enable new applications [1]. They represent a good candidate for DC-DC converters to be used in DC grids applications, such as future offshore wind farms collection networks [2-3]. SST also allows a better efficiency and a lower weight, which can be very

\author{
Marie-Ange Raulet \\ Univ Lyon, Université Claude Bernard Lyon 1, Ampère \\ F-69130 Villeurbanne, France \\ Email: marie-ange.raulet2@univ-lyon1.fr \\ Fabien Sixdenier \\ Univ Lyon, Université Claude Bernard Lyon 1, Ampère \\ F-69130 Villeurbanne, France \\ Email: fabien.sixdenier@univ-lyon1.fr
}

useful for offshore applications. The gain in performance obtained by the use of SST is mainly due to the possibility of increasing semiconductors switching frequency. It allows to use medium frequency transformers (MFT) which are more compact and more efficient than the traditional 50-60 Hz transformers [4]. Inside the converter, its functions are multiple: transformation ratio, galvanic insulation, specific values of inductances. Moreover, high power and high voltage applications combine insulation and cooling constraints on the MFT. Therefore, the MFT design must take into account all these specifications which represent a multi-objective optimization process [5].

Because of this complexity, a lot of work has been done to develop MFT design methodologies [6-11]. Most of these work have followed the strategy of calculating an important number of designs in order to cover the whole design space and select the best one against the specifications. Given the results obtained, this method has proven to be well adapted to MFT design. To calculate such a great number of designs, analytical or semianalytical models are extensively used, allowing a very fast calculation time. In order to choose the best design, the end user must have a certain confidence in the design tool and the models used in it. Consequently, these models must be as precise as possible and cover magnetic, electric and thermal aspects of the transformer. Each of the cited work proposes a combination of models to address critical points related to their specifications and the selected MFT structure.

However, papers of the literature always focuses on a specific design: the electrical specifications and the 
technological choices are fixed. The novelty of this paper is the fact that the method is as generic as possible. It is applicable to any electrical specifications and it covers a lot of the technological choices amongst the most common ones. Indeed, contrary to traditional lowfrequency transformers, the possible combinations of materials and technologies to manufacture MFT are numerous [12], and it is not obvious to decide which technological choices will be the best for a given specification. Therefore, a general methodology must be able to cover any combination of MFT geometry and materials. This means having either general models working in any case, or multiple models covering each case. This paper will either use models from literature if they are adapted, or use newly developed models if needed.

To this aim, Section II will detail the proposed MFT design methodology, the different technological choices and the associated models. Then, Section III will present a case study of the previously described methodology in order to follow a complete MFT design optimization for a specific MFT structure, including numerical Finite Element Method (FEM) validation of the selected design. Experimental validation could not be done because of the high cost of such prototype, and a small scale prototype would not really validate the calculations. Finally, Section IV will compare quantitatively different technological combinations for the same case study and analyze the impacts of those choices. It will also study the optimal operating frequency adapted to a specific structure.

\section{MFT Design Methodology}

\section{A. Design Flow Chart}

The general MFT design flow is described in Figure 1. It consists in three distinctive phases: pre-design, analytical design and design validation. Each phase has its own outputs and is quite independent of the others.

1) Pre-design: Pre-design phase purpose is to gather all the necessary inputs for the proper design to be performed. It includes voltages and currents magnitude and waveforms from the converter, insulation requirements according to standards, material properties and performance criteria. These criteria must define the accepted and/or targeted values for efficiency, volume, weight, inductances, capacitances and temperatures. In this phase, a qualitative analysis of the specifications and constraints can allow to select only the relevant technological choices.

2) Analytical Design: Then the analytical design phase consists in calculating a great number of designs based on the variations of the different degrees of freedom (as defined in II.C) related to the MFT structure. This phase leads to the selection of a MFT design matching the specifications. Equations and models that analytically calculate every aspect of the transformer are included in this phase. First, the dimensions of the transformer are computed from the degrees of freedom and the insulation distances. Then, inductances, capacitances and losses can be calculated with appropriate models. And finally, a thermal calculation is performed from the dimensions and the losses to determine the maximal temperatures in each active part. More information about the nature of models used will be given in II.D, II.E, and II.F.

3) Design Validation: Next step is the validation phase whose the goal is to ensure that the selected design will work properly. To do so, it must be verified with manufacturers that a design with calculated dimensions is feasible. In parallel, numerical simulations with FEM are performed to obtain more precise results on some critical aspects of the transformer. A circuit model of the MFT is also built up, from either analytical or numerical results, to be inserted in the converter simulation scheme and to ensure the right functioning of the complete converter.

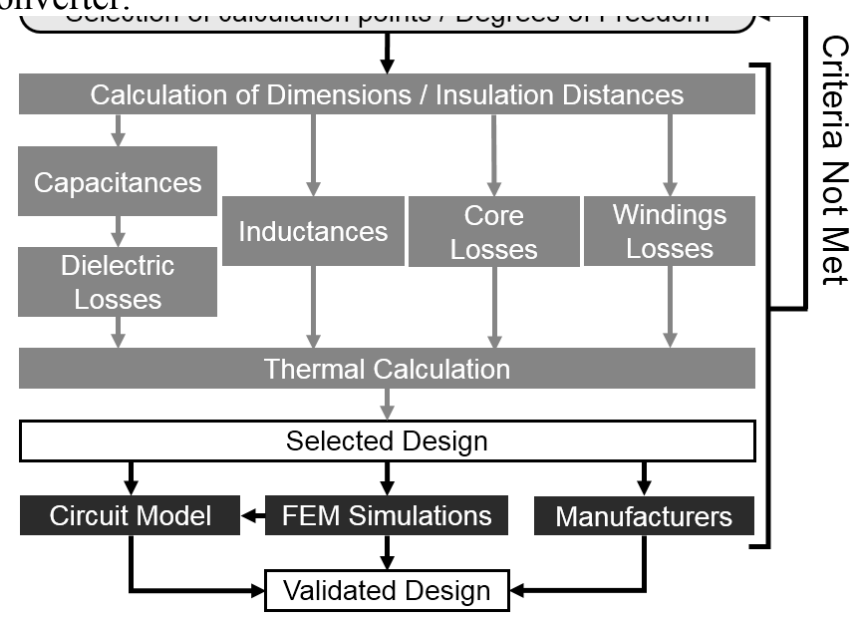

Fig. 1: Design Flow Chart showing pre-design steps (top-white), analytical design calculation (middle-light grey) and validation steps (bottom-black)

\section{B. Technological Choices}

A transformer structure is defined by a combination of magnetic core, windings, insulation, cooling and geometry. The Table 1 summarizes the existing technological choices for high power high voltage MFT, and compares the works from the literature with our approach in terms of which technological choices can be used for design. It can be seen that this work covers significantly more MFT structures than the state of the art.

Magnetic core is defined by its magnetic material. The choice of the material is closely linked to the frequency: $\mathrm{SiFe}$ sheets are the best candidate for low frequencies up to hundreds of $\mathrm{Hz}$, then comes amorphous followed by 
nanocrystalline for application up to some and a few tens of $\mathrm{kHz}$, and finally ferrites can go up to several tens of $\mathrm{kHz}$ [13-14].

TABle 1: MFT TeChNOLOGiCAL CHOICES

\begin{tabular}{|c|c|c|c|c|c|c|c|c|c|}
\hline \multicolumn{2}{|c|}{ Reference work } & [5] & [6] & [7] & [8] & [9] & [10] & [11] & This work \\
\hline \multirow{2}{*}{ Geometry } & Core-Type & & & & & & प & & प9 \\
\hline & Shell-Type & प1 & प्ष & प & प्ष & प & & पा & पा \\
\hline \multirow{4}{*}{$\begin{array}{c}\text { Magnetic } \\
\text { Core }\end{array}$} & SiFe sheets & & & & & & प & & प्ष \\
\hline & Amorphous & & & & प्ष & प्ष & & पा & 4 \\
\hline & Nanocrystalline & & प1 & & प्ष & प & & प1 & प \\
\hline & Ferrite & प1 & & & & & & & प्ष \\
\hline \multirow{3}{*}{ Windings } & Flat wire & & & & & & प & & \\
\hline & Litz wire & प & प्ष & प & प्ष & प & & प1 & प \\
\hline & Foil & & & & 4 & & प & & पा \\
\hline \multirow{4}{*}{ Insulation } & Air & प्ष & प्ष & प & प्ष & & प्व & प्ष & प \\
\hline & Air-Resin & & & & & & & & प \\
\hline & Resin & & & & & प & & & प5 \\
\hline & Oil & & & & & & & & प्ष \\
\hline \multirow{4}{*}{ Cooling } & Natural & प1 & प्ष & प & & & प & प्ष & प्ष \\
\hline & Forced & & & & & प & & & प \\
\hline & Radiator & & प्ष & $\Phi$ & & प & & & प1 \\
\hline & Cold Plates & & & & प & & & & $\Phi$ \\
\hline
\end{tabular}

Windings are also partly defined by their material (copper or aluminum in most cases), but more significantly by their internal structure. Typical conductors used for medium frequency applications are Litz cables and foils. However, continuously transposed cables may also be used for lower frequencies, and tubes can be an alternative if higher losses and liquid cooling system are not an issue.

The insulation mainly depends on the insulation voltage requirement. For low insulation voltage, air insulation is the most widely used, with resin impregnation or coating of windings if needed. But for higher insulation voltages, cast resin or oil insulation may be required.

Cooling of the transformer is typically done by natural or forced convection on the external surfaces with the available fluid (air or oil). More integrated solutions can also make use of cold plates on specific faces of the transformer to either improve the cooling performance or reduce the transformer volume [15]. However, this solution also requires an external liquid cooling system that may increase the total volume and losses of the solution.

Finally, the geometry of the transformer is the way to arrange windings and core together. For single-phase transformers, most common structures are core-type and shell-type, as shown in Figure 2. These are the only geometries that will be considered in this paper. However, there are other geometries such as toroidal core [16], coaxial windings [17] or matrix core configuration [18].
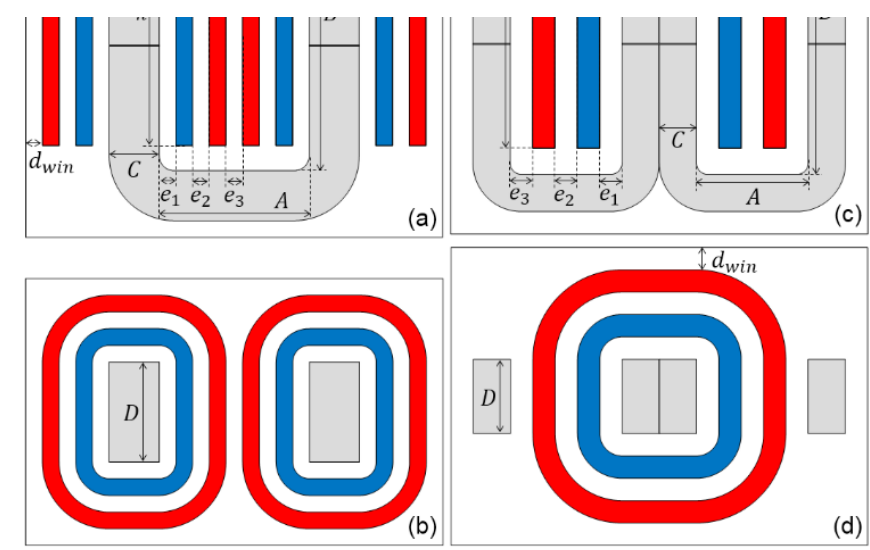

Fig. 2: Geometry schematics for (a) Core-Type front view, (b) CoreType top view, (c) Shell-Type front view, and (d) Shell-Type top view in case of air insulation

\section{Geometry Calculation and Degrees of Freedom}

The dimensions for a specific geometry can be determined from electrical inputs and a set of basic physical and geometric equations. The main physical equation is derived from Faraday's law of induction (1) and establishes the relation between voltage $V$, number of turns $N$, magnetic cross-section $S_{\operatorname{mag}}$ and maximal induction $B_{\max }$ as shown in (2). This is the general formula for any voltage waveform, as long as the voltage is periodical with a mean value of zero. This formula reduces to (3) if the voltage is sinusoidal and (4) if the voltage is square, in which $f$ is the frequency. These equations are valid either for primary or secondary winding. The relation between primary and secondary number of turns is the classical one of transformers (5).

$$
\begin{aligned}
& V(t)=-N \frac{d \varphi(t)}{d t}=-N S_{\text {mag }} \frac{d B(t)}{d t} \\
& N S_{\text {mag }} B_{\text {max }}=\max \left(\int V(t) d t\right) \\
& V_{R M S}=4.44 f N S_{\text {mag }} B_{\text {max }} \\
& V_{R M S}=4 f N S_{\text {mag }} B_{\text {max }} \\
& V_{1} / V_{2}=N_{1} / N_{2}
\end{aligned}
$$

A first geometric equation is the relation between magnetic cross-section $S_{m a g}$, core filling factor $\eta_{m a g}$ and core dimensions (6). Then, conductor cross-section $\mathrm{S} 1$ is determined from current density $j_{l}$ and associated current $I_{l}$. For Litz windings, it is also related to number of strands $N_{s l}$ and strand diameter $d_{s l}$ (7), while for foil windings the foil thickness $f_{l}$ and winding height $w_{h}$ are used (8). Windings dimensions can be linked with the number of turns $N_{l}$, the conductor cross-section $S_{l}$ and the winding filling factor $\eta_{1}(9)$. This filling factor represents the proportion of conductive material inside the space occupied by a winding. These three equations exists for 
both primary and secondary windings. Finally, two form factors are defined for the winding window (10) and for the magnetic core cross-section (11). All the variables in the equations correspond to the ones described in Figure 2.

$$
\begin{aligned}
& S_{\text {mag }}=S_{\text {core }} \eta_{\text {mag }}=C D \eta_{\text {mag }} \\
& S_{1}=\frac{I_{1}}{j_{1}}=\frac{\pi}{4} N_{s 1} d_{s 1}^{2} \\
& S_{1}=\frac{I_{1}}{j_{1}}=w_{h} f_{1} \\
& N_{1} S_{1}=w_{1} w_{h} \eta_{1} \\
& F_{\text {win }}=B / A \\
& F_{\text {mag }}=C / D
\end{aligned}
$$

Some variables of these equations must be fixed to be able to find a solution, and they will constitute the degrees of freedom of the problem. The degrees of freedom that were selected are maximal induction $B_{\max }$, current densities $j_{l}$ and $j_{2}$, primary number of turns $N_{l}$, strand diameters $d_{s 1}$ and $d_{s 2}$ for Litz windings or foil thicknesses $f_{1}$ and $f_{2}$ for foil windings, and form factors $F_{\text {win }}$ and $F_{\text {mag. }}$. Then, for each combination of degrees of freedom, it is possible to calculate a transformer geometry and therefore the each part's volumes and weights of each part. It should be noted that insulation distances and resin thicknesses (in case of Air-Resin or Resin insulation) are fixed values, defined from dielectric withstand and mechanical integration considerations. Those values could be adapted if the dielectric losses are too important.

\section{Electrical Equivalent Circuit}

Once the geometry of the transformer is known, the electrical parameters as shown in Figure 3 can be calculated.

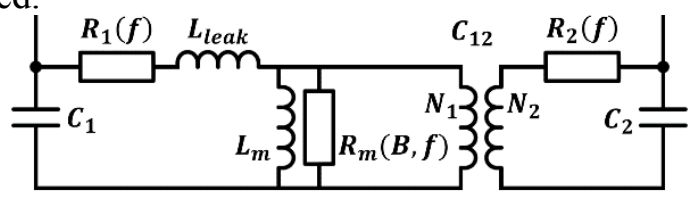

Fig. 3: Electrical equivalent circuit of a transformer with inductances, losses resistances and parasitic capacitances.

1) Magnetizing and Leakage Inductances: Under linear conditions, the magnetizing inductance is obtained from a simple reluctance calculation, considering the magnetic cross-section $S_{m a g}$, the magnetic path length $l_{\text {mag }}$, the cumulated air gap thickness $g$, the magnetic material permeability $\mu_{\text {mag }}$ and the core cross-section $S_{\text {core }}(12)$.

$$
L_{m}=\frac{N_{1}^{2}}{\frac{l_{m}}{S_{\text {mag }} \mu_{\text {mag }}}+\frac{g}{S_{\text {core }} \mu_{0}}}
$$

The leakage inductance is calculated with the hypothesis of a mono-dimensional field, even though this hypothesis is rarely true in transformers, as described in [19]. However, an improvement of this formula (13) described in [20] allows to keep a fast calculation time while improving the accuracy in the specific case of the transformer. In this equation, $K_{R}$ is the Rogowski factor used to correct the leakage field length along the winding axis, while $l_{\text {leak }}$ is the meanlength of the leakage layer.

$$
\begin{aligned}
& L_{\text {leak }}=\frac{N_{1}^{2} \mu_{0} l_{\text {leak }}}{\frac{w_{h}}{K_{R}}}\left(\frac{w_{1}}{3}+e_{2}+\frac{w_{2}}{3}\right) \\
& K_{R}=1-\left(1-\exp \left(-\frac{\pi w_{h}}{w_{1}+e_{2}+w_{2}}\right)\right) \times \frac{w_{1}+e_{2}+w_{2}}{\pi w_{h}}
\end{aligned}
$$

2) Parasitic Capacitances: The parasitic capacitance between primary and secondary windings $C_{12}$ could be calculated using the typical formula for parallel plate capacitor. However, the ratio between winding height $w_{h}$ and distance between windings $e_{2}$ may be too low in some cases for the formula to remain valid: edge effects can have a significant impact on the value of the capacitance. This is why a calculation based on electric field integration has been performed to include edge effects in the capacitance calculation. Based on FEM simulation analysis, the electric field pattern at the edges of a plane capacitor has been considered as shown in Figure 4.

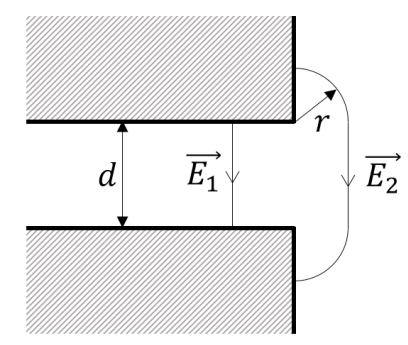

(a)

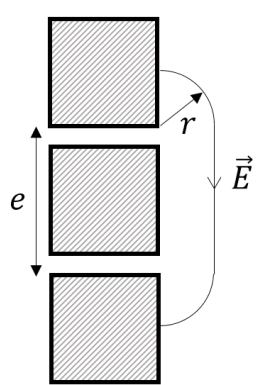

(b)
Fig. 4: Schematic of the considered electric field pattern for the calculation of parasitic capacitances for (a) consecutive turns, and (b) non-consecutive turns

For a voltage difference $V$ between the two conductors, the electric field can be expressed with (14). Then, the electric field is integrated to obtain the stored energy (15), and finally the capacitance value (16). $\varepsilon$ is the permittivity of the insulating material, $w$ is the width of the surfaces in regards, $l$ is the depth of the considered 2D geometry and $r_{\max }$ is the limit of integration. This limit is a function of the dimension of conductors perpendicularly to the opposing surfaces. 


$$
\begin{aligned}
& E_{1}=\frac{V}{d} \\
& E_{2}=\frac{V}{d+\pi r} \\
& W_{\text {elec }}=\frac{1}{2} C V^{2}=\frac{\varepsilon}{2} \iiint_{\% \text { MEGA }} E^{2} d \% \text { OMEGA } \\
& C=\frac{\varepsilon w l}{d}+2 \frac{\varepsilon l}{\pi} \ln \left(\frac{\pi r_{\text {max }}+d}{d}\right)
\end{aligned}
$$

For Litz windings, laminated Litz cables with almost rectangular or square cross-section are considered because of their higher filling factor. Therefore the capacitances between consecutive turns $C_{\text {cons }}$ can also be calculated using (16). However, in some cases the capacitances between non-consecutive turns $C_{n \text {-cons }}$ cannot be neglected because the distance between them is short. In this case, these capacitances can be calculated by integrating only electric field on the edges of nonconsecutives turns of the same layers. In (17), $e$ is the distance between the considered turns.

$$
C_{n-\text { cons }}=2 \frac{\varepsilon l}{\pi} \ln \left(\frac{\pi r_{\max }+e}{e}\right)
$$

Finally, the total equivalent self-capacitance of the Litz winding is calculated by summing the energies stored by each elementary capacitance and normalizing this energy with regards to the winding total voltage (18). In the following expression, $i$ is the number of turns between the two non-consecutive turns considered and $N$ is the total number of turns of the winding.

$$
\begin{aligned}
W_{\text {total }} & =\frac{N-1}{2} C_{\text {cons }} \times 1^{2}+\sum_{i=1}^{N-2} \frac{N-1-i}{2} C_{n-\text { cons }}(i) \times(i+1)^{2} \\
C_{\text {total }} & =\frac{2 W_{\text {total }}}{(N-1)^{2}}
\end{aligned}
$$

In the case of foil windings, the capacitance between two turns is considered to be a plane capacitor with no edge effects, because there is a high ratio between foil height and insulation thickness between consecutive foils. It also means that parasitic capacitances of foil windings will be higher than the ones of Litz windings in general. Therefore, the problem is to take into account correctly the multilayer structure of the foil winding. It can be done using (19) which has been demonstrated in [21], where $n$ is the considered layer, $l_{n}$ the mean-length associated to this layer and $d_{f}$ the distance between two consecutive foil layer.

$$
C_{\text {foil }}=\sum_{n=1}^{N-1} C_{n}\left(\frac{2}{n}\right)^{2}=\sum_{n=1}^{N-1} \frac{\varepsilon w_{h} l_{n}}{d_{f}}\left(\frac{2}{n}\right)^{2}
$$

All the reactive components of the transformer electrical equivalent scheme can now be calculated. Resistive components are calculated from losses, which will be seen in the next part.

\section{E. Losses Calculation}

1) Core Losses: The Steinmetz's equation [22] allows to easily calculate magnetic losses for design purposes. It expresses the dependence of magnetic losses to maximal induction $B_{\max }$ and frequency $f$, while assuming that these dependences follow a power law (20). This hypothesis remains valid for a limited range of frequency and induction for which the Steinmetz parameters $k, a$ and $b$ can be calculated. However, this formula is only valid for sinusoidal induction, which is in general not the case for MFT. A lot of work has been done to adapt the Steinmetz's equation to non-sinusoidal induction while using the same parameters. In [23], a comparison of different modified versions of Steinmetz formula is performed. It appears that IGSE (Improved generalized Steinmetz Equation) [24] is one of the best performing model, and it can be applied to any waveform. That is why IGSE (21) will be used to calculate magnetic losses. Calculated losses can either be volumetric or specific losses, depending on the value of $k$ parameter.

$$
\begin{aligned}
& P_{\text {mag }}=k \times f^{a} \times B_{\text {max }}^{b} \\
& P_{\text {mag }}=\frac{1}{T} \int_{0}^{T} \frac{k\left(\left.\Delta B\right|^{b-a}\right.}{(2 \pi)^{a-1} \int_{0}^{2 \pi}|\cos \theta|^{a} 2^{b-a} d \theta}\left|\frac{d B(t)^{a}}{d t}\right|^{d} d t
\end{aligned}
$$

2) Windings Losses: A complete analysis of various available models for taking into account frequencydependent resistance in transformers has been performed in [19]. For Litz windings, it appears that models based on the formula established by Albach in [25] are more robust than others. To keep a low computation time, this model must be used with a 1D magnetic field hypothesis. In this case it can be simplified in an analytical formula (22) without numerical discretization, where $\delta$ is the skin depth, $a$ is the strand diameter, $\eta$ is the winding filling factor, $N$ is the number of turns, $w$ is the winding width, $h$ is the winding height and $I_{n}$ are the modified Bessel functions of the first kind.

$$
\begin{aligned}
& \frac{R_{A C}}{R_{D C}}=\frac{1}{2} \operatorname{Re}\left(\alpha a \frac{I_{0}(\alpha a)}{I_{1}(\alpha a)}\right)+\frac{4}{3} \frac{\eta \pi N w}{h} \operatorname{Re}\left(\alpha a \frac{I_{1}(\alpha a)}{I_{0}(\alpha a)}\right) \\
& \alpha=\frac{1+i}{\delta}
\end{aligned}
$$


For foil windings, Dowell's model [26] is particularly adapted because it supposes windings composed of successive rectangular-shaped conductive layers of thickness $d$. The formula (23) expresses the resistance increase compared to DC resistance in function of skin depth $\delta$, number of layers $m$, foil thickness $d$ and the ratio between winding height $w_{h}$ and window height $B$.

$$
\begin{aligned}
& \frac{R_{A C}}{R_{D C}}=X^{\prime}\left(\frac{\sinh \left(2 X^{\prime}\right)+\sin \left(2 X^{\prime}\right)}{\cosh \left(2 X^{\prime}\right)-\cos \left(2 X^{\prime}\right)}+\frac{2}{3}\left(m^{2}-1\right) \frac{\sinh X^{\prime}-\sin X^{\prime}}{\cosh X^{\prime}+\cos X^{\prime}}\right) \\
& X^{\prime}=\frac{d}{\delta} \sqrt{\frac{w_{h}}{B}}
\end{aligned}
$$

3) Dielectric Losses: Insulating materials can have a significant loss tangent $\tan (\delta)$, which combined with the medium frequency voltage can lead to significant dielectric losses. Most of the time, the voltages applied to parasitic capacitances are not sinusoidal and have an important harmonic content. Calculation of dielectric losses must be performed by summing the losses associated to each voltage harmonic component as shown in (24). This formula is used to calculate dielectric losses for both self-capacitances $C_{1}$ and $C_{2}$, and primary to secondary capacitance $C_{12}$.

$$
P=\sum_{f} 2 \pi f C \tan (\delta(f)) \frac{V_{\text {peak }}^{2}(f)}{2}
$$

\section{F. Thermal Model}

A classical approach is used, with a thermal network based on the electrical-thermal analogy. Losses are modelled by power sources while heat transfers are modelled by thermal resistances, and each node of the thermal network has its own temperature. The number of nodes is a crucial choice: with too few nodes, the accuracy of the thermal network and its ability to estimate maximal temperature in each material might be compromised, while with too many nodes, the resolution of the electrical equivalent network becomes too complex and too time-consuming.

1) Conduction: To calculate conduction resistances, the classical formula (25) based on the thermal conductivity $k$ of the material and its dimensions ( $l$ is the length and $S$ is the cross-section) can be used. However, to use such a conduction thermal resistance, a preferred direction of heat must be identified. Furthermore, this formula is only valid in a domain where conductive heat transfer is predominant and there is no other heat source inside the domain. If the domain is subject to a homogeneous heat generation rate per volume unit, the resolution of heat equation leads to another formula (26) where the conduction thermal resistance must be divided by two if the heat source is localized at the supposed hotspot, as demonstrated in [27]. Therefore, this last formula must be used to calculate the conduction resistance inside the windings and inside the magnetic core. Other parts, such as coil former, are not considered because they are not defined at this stage of the design.

$$
\begin{aligned}
& R_{\text {th-cond }}=\frac{l}{k S} \\
& R_{\text {th-cond-source }}=\frac{l}{2 k S}
\end{aligned}
$$

For bulk materials, such as ferrite or cast resin, the thermal conductivity is isotropic. However, for more complex structures such as tape-wound cores, foil windings or Litz windings, the equivalent thermal conductivity is anisotropic and is a function of the arrangement and the thermal conductivities of materials. For a succession of two different materials layers, longitudinal (parallel to layers) and transverse (normal to layers) thermal conductivities can be calculated according to (27) and (28), where $k_{1}$ and $k_{2}$ are the thermal conductivities of the two different materials, and $\eta_{1}$ and $\eta_{2}$ are the material proportions. These formulas are used in the case of foils, tape-wound cores and steel sheets cores.

$$
\begin{aligned}
& k_{l}=\eta_{1} k_{1}+\eta_{2} k_{2} \\
& k_{t}=\frac{k_{1} k_{2}}{\eta_{1} k_{2}+\eta_{2} k_{1}}
\end{aligned}
$$

For Litz windings, the arrangement of strands, enamel and gap between strands must be considered. A model has been developed in [28]. The longitudinal thermal conductivity is an area-weighted average of the different material conductivities. The transverse thermal conductivity is calculated with specific paths of integration associated with infinitesimal thermal resistances, considering either square-packed wires or hexagonal-packed wires. In Litz cables with a high number of strands, the strands are more randomly organized but it can be considered to be square-packed and hexagonal-packed in equivalent proportion, and so the thermal conductivity can be seen as an average of the two cases.

2) Convection: The complexity of calculating convection resistances comes from the difficult evaluation of the convection coefficient value. However, there exist empirical formulas based on experimental results for typical surfaces [29-30]. The Table 7 (in Appendix A) lists the different types of faces that are considered for the transformer geometry and how the convection coefficient can be calculated for this type of face.

3) Radiation: Radiation thermal resistances can be calculated from Stefan-Boltzmann law (29), knowing the 
emissivity $\varepsilon$, the Stefan-Boltzmann constant $\sigma$, the surface $S$, the ambient temperature $T_{a m b}$ and the temperature of the considered surface $T$. In transformer geometries, radiation is only considered for surfaces facing outwards and is usually neglected between transformer parts.

$$
R_{t h-r a d}=\frac{\Delta T}{P_{\text {rad }}}=\frac{\left(T-T_{\infty}\right)}{\varepsilon \sigma S\left(T^{4}-T_{\infty}^{4}\right)}
$$

4) Thermal Network Resolution: The next step to establish the thermal network is to geometrically place the nodes of the network. A node must be present at each potential hotspot, and also at each surface because surfaces temperatures are required for convection and radiation calculation. The considered nodes of the thermal network for Core-Type geometry with air insulation are showed on Figure 5. Nodes for Shell-Type geometry are placed following the same pattern. In case of resin or air-resin insulation, there are additional nodes at the interfaces between windings and resin. Heat sources are localized on the potential hotspots nodes, with a value corresponding to the integration of loss density in a surrounding virtual block. Even though potential hotspots nodes are drawn at the centre of core cross-section and windings cross-sections, it may not be the case in reality. In fact, the position of the hotspot in the cross-section will depend on the surfaces temperatures as demonstrated in [27]. In (30), $k$ is the thermal conductivity, $q$ is the heat generation rate per volume unit, $l$ is the distance between the surfaces, $T_{l}$ is the temperature at the first surface $(x=0), T_{2}$ is the temperature at the second surface $(x=l)$ and $x_{\max }$ is the hotspot location.

$$
x_{\max }=\frac{l}{2}+\left(T_{2}-T_{1}\right) \frac{k}{l q}
$$

Therefore, conduction, convection and radiation thermal resistances depend on surface temperatures, which are initially unknown. This is why an iterative resolution process must be performed to calculate all temperature-dependent thermal resistances. Temperatures at each node are calculated from the values of thermal resistances and heat sources, at each iteration. To solve this electrical equivalent network, numerical methods using admittance matrix inversion exist [31]. However, the electrical equivalent network is generally not too complex and can also be solved analytically using Kirchhoff's circuit laws and Ohm's law. Typically, it takes between five to ten iterations to converge. After convergence, maximal temperatures in each material can be easily obtained and compared with the maximal admissible temperature, thus allowing to keep or to exclude a design.

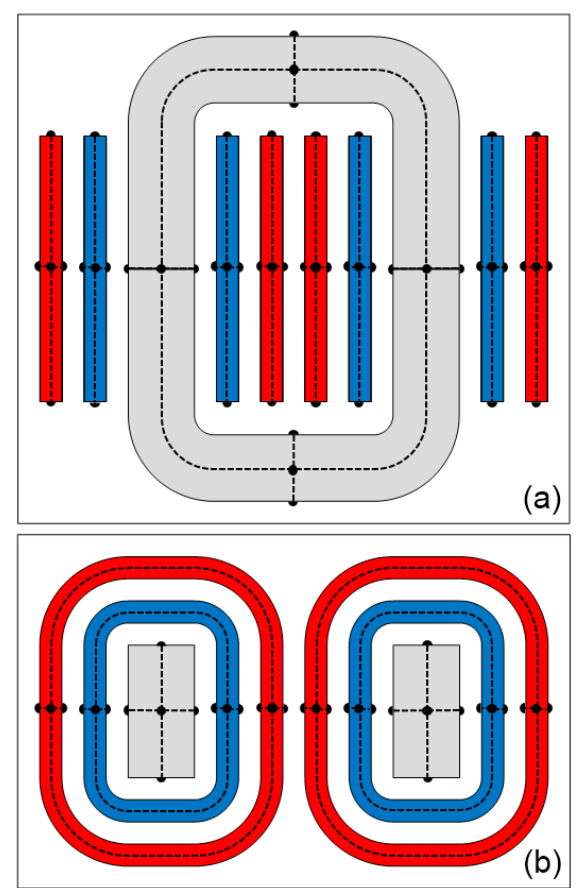

Fig. 5: Nodes of the thermal network for Core-Type geometry with air insulation (a) front view and (b) top view. Full circles represent potential hotspots nodes, half circles represents surface temperature nodes and dashed lines represent conduction paths

\section{CASE StUdY}

\section{A. Definition of the Application}

The case study considered is a DC-DC converter with a modular topology as described in [32], for a multimegawatt converter in offshore windfarm application.

TABle 2: MFT SPECIFICATIONS \& TeChNOLOGiCAl ChOices

\begin{tabular}{|c|c|}
\hline Apparent Power & $1 \mathrm{MVA}$ \\
\hline Nominal Power & $0.8 \mathrm{MW}$ \\
\hline Frequency & $20 \mathrm{kHz}$ \\
\hline Voltages & $4.4 \mathrm{kV}: 4.4 \mathrm{kV}$ \\
\hline Currents & $240 \mathrm{~A}: 240 \mathrm{~A}$ \\
\hline Electrical Insulation & $80 \mathrm{kV} \mathrm{DC}$ \\
\hline Leakage inductance & $30 \mu \mathrm{H}<L_{\text {leak }}<80 \mu \mathrm{H}$ \\
\hline Efficiency & $>99 \%$ \\
\hline Geometry & Core-Type \\
\hline Magnetic Core & Nanocrystalline Cut-Cores VITROPERM $500 \mathrm{~F}$ \\
\hline Windings & Copper Litz cables \\
\hline Insulation & Oil MIDEL 7131 synthetic ester \\
\hline Cooling & Forced Convection - Oil temperature $40^{\circ} \mathrm{C}$ \\
\hline
\end{tabular}

The Table 2 summarizes the electrical specifications and the firstly considered technological choices for the transformer. Moreover, the waveforms of voltages and currents considered for sizing are available in Figure 6. There are no specific constraints on weight, magnetizing inductance and parasitic capacitances. With all these information, the pre-design steps are now complete and the analytical design can be started. 


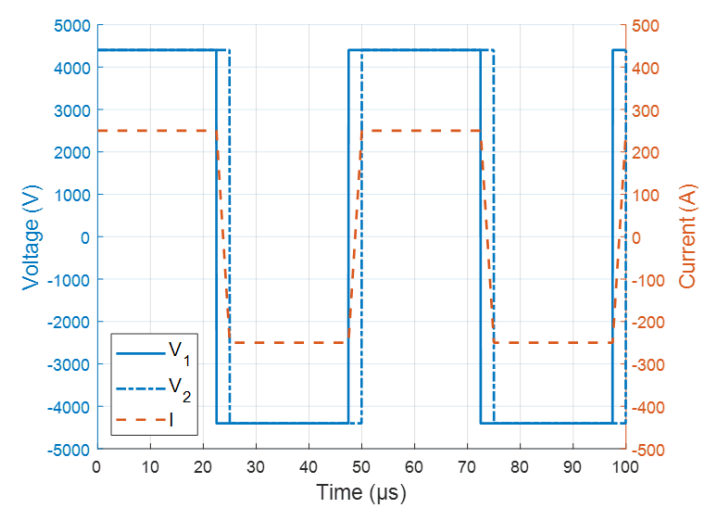

Fig. 6: Voltages and current waveforms applied to the transformer, the current is the same in both primary and secondary windings

\section{B. Analytical Design}

The first step of the analytical design is to gather the properties for each material: density, electrical conductivities, permittivity, dissipation factor, permeability, Steinmetz parameters, thermal conductivities, specific heat capacity, filling factors and maximum temperatures. Most of these data come from datasheet of manufacturers, but some of them such as the Steinmetz parameters were obtained from characterizations. Then, insulation distances between each part of the transformer are defined from the dielectric strength of the insulating material, taking into account an effective dielectric strength with a safety factor. For the oil used, it results in $20 \mathrm{~mm}$ insulation distance to withstand $80 \mathrm{kV}$ DC.

The different degrees of freedom have been swept to cover the design space properly, and the calculation took $72 \mathrm{~s}$ for 1.5 million of designs, on a laptop with quad core CPU $2.4 \mathrm{GHz}$ and $8 \mathrm{~GB}$ RAM, which is a common and accessible computing equipment. Filters were applied to the raw results to discard designs with too high temperatures. In this case, maximal allowed temperatures are $120^{\circ} \mathrm{C}$ for both core and windings. The results of the analytical calculations are displayed in Figure 7. On this figure, the compromise between efficiency and volume can clearly be seen. All the points corresponding to a maximum efficiency for a given volume draw a Pareto front. It can also be seen that most compact designs have higher maximal temperature, as expected, reaching the limit value of $120^{\circ} \mathrm{C}$.

The Table 3 compares the performances of the optimization algorithm from literature and this work. It can be seen than most of the time dielectric aspects are not taken into account in other papers. Optimization methods are either genetic algorithm (GA), brute-force $(\mathrm{BF})$, mesh refinement (MR) or manual (M). In terms of calculation time per design point, the method proposed here seems to be several orders of magnitude faster than the ones from literature, even if data is not always available forin alleach references. Also, when used, and the calculation time is dependent on hardware.

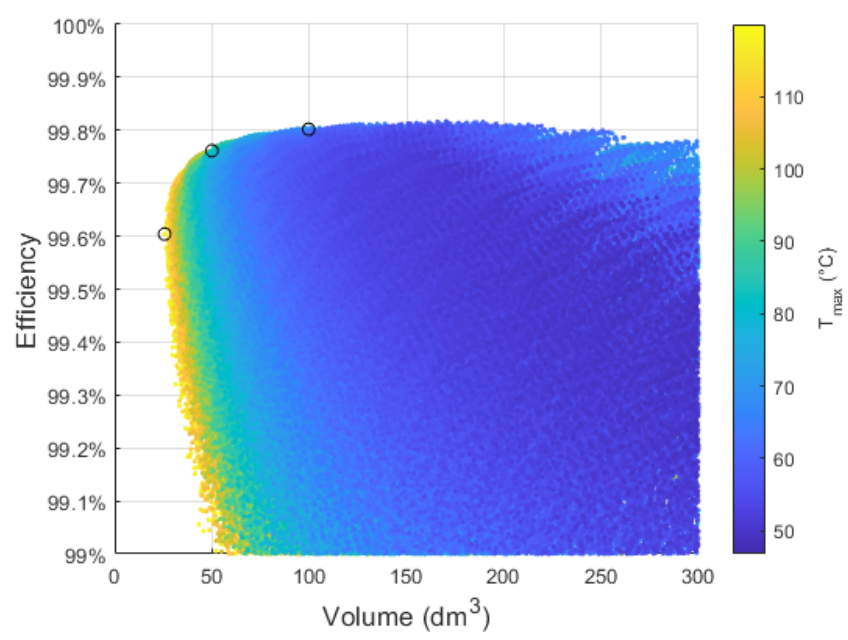

Fig. 7: Efficiency, volume and maximal temperatures of 1.5 million design points.

TABle 3: PerformanCes of OPTIMIZATION ALGORITHMS

\begin{tabular}{|c|c|c|c|c|c|c|c|c|}
\hline Reference work & [5] & [6] & [7] & [8] & [9] & [10] & {$[11]$} & This work \\
\hline Leakage Ind. & प & प & t & & tg & t & t9 & $\Phi$ \\
\hline Magnetizing Ind. & प् & & प्र & & & & प्ष & प \\
\hline Core Losses & प & $\Phi$ & $\square$ & $\square$ & प & प & प & 甲 \\
\hline Windings Losses & प & प & 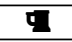 & प्9 & 모 & पㄹ & 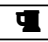 & 호 \\
\hline Parasitic Capacitances & & & & & & & & tq \\
\hline Dielectric Losses & & & & & & & & 모 \\
\hline Temperatures & प9 & $\Phi$ & $\square$ & प & प & प & प & t9 \\
\hline Optimization Method & GA & $\mathrm{BF}$ & $\mathrm{BF}$ & MR & GA & $\mathrm{M}$ & $\mathrm{M}$ & $\mathrm{BF}$ \\
\hline Total time (s) & 180 & & 10000 & 1800 & & & & 72 \\
\hline No. points (thousands) & 40 & 400 & 2000 & & & & & 1500 \\
\hline Time / design (ms) & 4.5 & & 5 & & & & & 0.05 \\
\hline
\end{tabular}

In our case, the design with the minimum volume and an efficiency above $99 \%$ (according to specifications) will be retained as the optimal design, because a lower volume is beneficial for integration and cost. Its detailed geometry is shown in Figure 8 and a summary of its main characteristics is availableare summarized in Table 4.

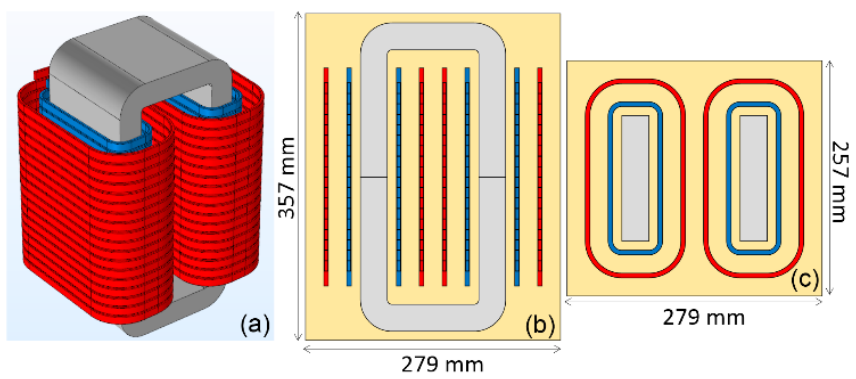

Fig. 8: Most compact design with Core-Type geometry, Nanocrystalline core, Litz cables, Oil insulation and cooling. (a) 3D Isometric view, (b) Front view, (c) Top view 
TABLE 4: MFT CHARACTERISTICS

\begin{tabular}{|c|c|c|c|}
\hline Characteristic & Analytical Value & FEM Value & $\begin{array}{c}\text { Relative } \\
\text { Difference }\end{array}$ \\
\hline Maximal induction & $0.504 \mathrm{~T}$ & - & - \\
\hline Number of turns & $36: 36$ & - & - \\
\hline Current density & $9.58 \mathrm{~A} / \mathrm{mm}^{2}$ & - & - \\
\hline Strand number and diameter & $3207 \mathrm{x} 0.1 \mathrm{~mm}$ & - & - \\
\hline Volume (box) & $25.6 \mathrm{dm}{ }^{3}$ & - & - \\
\hline Weight Core & $18.7 \mathrm{~kg}$ & - & - \\
\hline Weight Windings & $7.9 \mathrm{~kg}$ & - & - \\
\hline Weight Oil & $18.6 \mathrm{~kg}$ & - & - \\
\hline Total Weight & $45.2 \mathrm{~kg}$ & - & - \\
\hline Efficiency & $99.60 \%$ & $99.61 \%$ & $+0.97 \%$ \\
\hline Core Losses & $831 \mathrm{~W}$ & $812 \mathrm{~W}$ & $+2.4 \%$ \\
\hline Windings Losses & $2183 \mathrm{~W}$ & $2179 \mathrm{~W}$ & $+0.19 \%$ \\
\hline Dielectric Losses & $166 \mathrm{~W}$ & $159 \mathrm{~W}$ & $+4.5 \%$ \\
\hline Max. Core Temperature & $118^{\circ} \mathrm{C}$ & $126^{\circ} \mathrm{C}$ & $-10.0 \%$ \\
\hline Max. Windings Temperature & $116^{\circ} \mathrm{C}$ & $117^{\circ} \mathrm{C}$ & $-1.4 \%$ \\
\hline Magnetizing Inductance & $14.4 \mathrm{mH}$ & $15.1 \mathrm{mH}$ & $-4.7 \%$ \\
\hline Leakage Inductance & $37.5 \mu \mathrm{H}$ & $35.9 \mu \mathrm{H}$ & $+4.5 \%$ \\
\hline Primary-Secondary Capacitance & $445 \mathrm{pF}$ & $424 \mathrm{pF}$ & $+5.0 \%$ \\
\hline Primary Capacitance & $23 \mathrm{pF}$ & $32 \mathrm{pF}$ & $-29.9 \%$ \\
\hline Secondary Capacitance & $32 \mathrm{pF}$ & $33 \mathrm{pF}$ & $-4.6 \%$ \\
\hline
\end{tabular}

\section{Numerical Validation}

1) Inductances and Capacitances: Inductances are validated with a 3D Magnetostatic FEM study. Magnetic core, air gap and homogenized windings are considered. With this method, a magnetizing inductance of $15.1 \mathrm{mH}$ and a leakage inductance of $35.9 \mu \mathrm{H}$ were obtained. These values are in good agreement with the analytical ones with less than 5\% deviation, and confirm the validity of the analytical models used. This is true because the designs have a low air gap value of $200 \mu \mathrm{m}$ per gap (typical parasitic air gap value of nanocrystalline cut cores), and so fringing effect is limited. It is important that this condition is met to avoid additional losses due to fringing effect, in both magnetic core and windings.

The capacitance between primary and secondary windings was obtained with the same geometry (homogenized windings) and FEM software, but using an electrostatic solver. A parasitic capacitance of $424 \mathrm{pF}$ was obtained, with again less than $5 \%$ deviation compared to the capacitance of $445 \mathrm{pF}$ obtained analytically with (16). If edge effects were not taken into account, an analytical value of $414 \mathrm{pF}$ would have been obtained. Therefore in this case, edge effects have a small impact on the parasitic capacitance value.

For parasitic capacitances of primary and secondary windings, the detailed geometry of windings must be considered. Therefore, 36 short-circuit turns for both primary and secondary windings were modelled. Parasitic capacitances of $32.5 \mathrm{pF}$ and $33.0 \mathrm{pF}$ were obtained with FEM simulations, respectively for primary and secondary windings. The difference is below $5 \%$ for secondary windings whereas it goes up to almost $30 \%$ for the primary windings. It shows that the model developed for parasitic capacitance of laminated cables is only able to give a rough estimation in this case. However, the value of these capacitances is usually not a critical parameter in medium frequency transformer design so a rough estimation is acceptable. This deviation can be explained by the presence of magnetic core next to the primary winding which modifies the shape of electric field in this area and therefore the values of non-consecutives turns parasitic capacitances (17). If edge effects and non-consecutive turns capacitances were neglected, the parasitic capacitances values obtained analytically would be $8.8 \mathrm{pF}$ and $12 \mathrm{pF}$ for primary and secondary windings, corresponding to an underestimation by three times of the parasitic capacitances. It shows that the developed model (18) increases significantly the accuracy in this case.

2) Losses: Magnetic losses cannot be directly and easily validated by FEM simulations from Maxwell equations because of the nature of static and dynamic hysteresis inside magnetic materials. An estimation of the loss density with IGSE (21) was performed by posttreating the results of a $3 \mathrm{D}$ magnetostatic simulation. The advantage of this method is that it does not require additional parameters, however it is only valid in the area where the magnetic material is used in its quasilinear domain. By doing so, total magnetic losses of $812 \mathrm{~W}$ were obtained numerically, which is very close to the value of $831 \mathrm{~W}$ obtained analytically. The difference here can be explained by the non-homogeneous level of induction in the corners of the magnetic core which is not considered analytically.

Validation of windings losses is more about determining numerically the additional losses due to skin and proximity effects rather than to validate the DC Joules losses. Therefore, magnetoharmonics FEM simulations have to be performed. However, modelling a Litz cable is complex and this is why a $2 \mathrm{D}$ simulation was considered. The geometry is the winding window, because this is where the magnetic field is the most confined and therefore where the proximity effects will be the most important. The magnetic core acts as a symmetry plane for the magnetic field [19], and due to the symmetry of this geometry, only a quarter of the winding window is modelled. In our case, there are 3207 strands per turn and a quarter of the winding window would therefore include more than 50000 strands. Meshing all the details of this kind of geometry is far too complex. This is why an approach using homogenized cables for all turns except one has been used. Skin and proximity effects are only solved for this turn, while the other turns are present only to generate a correct "environment" magnetic field. Figure 9 shows the geometry used where it can be seen that the secondary winding is homogenized whereas the primary turns are not. Amongst the primary turns, only the ones located at the middle and at the bottom of the winding were modelled in details with their strands structure. In fact, the magnetic field cartography is really different around the turns located at the bottom of the winding 
because this is where edge effects were expected and consequently proximity effects are different.

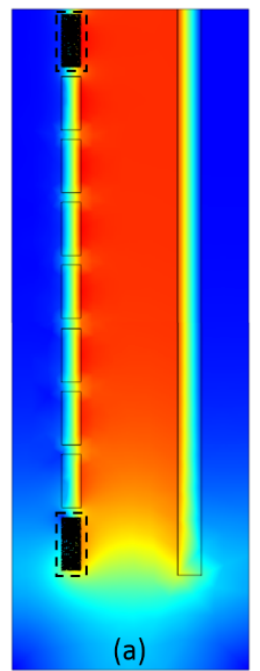

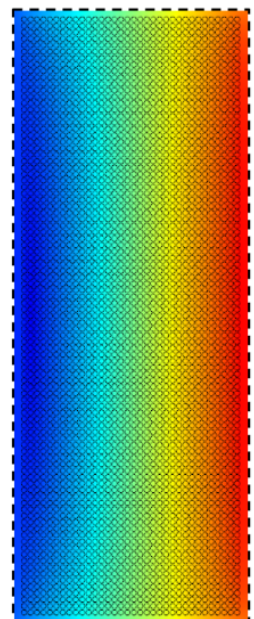

(b)

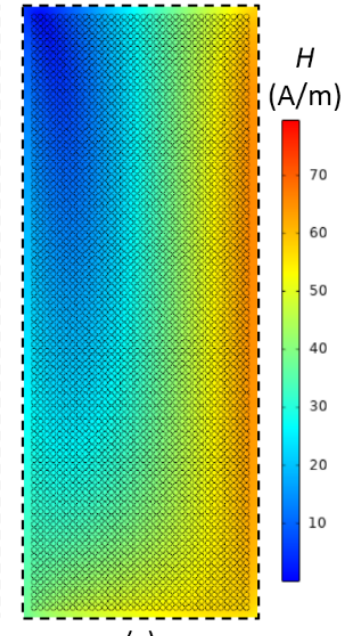

(c)
Fig. 9: Magnetic field cartography obtained with 2DFEM Magnetoharmonic simulation. (a) A quarter of the winding window. (b) Turn located at the middle of the winding. (c) Turn located at the bottom of the winding.

This simulation was performed for various frequencies, corresponding to the current harmonics up to $1 \mathrm{MHz}$. For each frequency, the resistance elevation factor was calculated for both turns located in the middle and the bottom of the winding. The results are displayed in Figure 10 where it can be seen that the chosen analytical model matches correctly the results obtained for the turn in the middle of the winding, with less than $1 \%$ deviation over the whole frequency range considered. However, for the bottom turn, the analytical model overestimates the resistance elevation factor by up to $12 \%$ at $100 \mathrm{kHz}$ and $30 \%$ at $1 \mathrm{MHz}$. Considering the resistance elevation factors obtained numerically and taking into account the edge effects, the global resistance elevation factor has been calculated for the distribution of harmonics corresponding to the application. An elevation factor of 1.139 was obtained with numerical results whereas analytical model gives 1.141 . This is less than $1 \%$ deviation on the estimation of additional losses, and the deviation on windings losses is even lower, with total windings losses of $2179 \mathrm{~W}$ obtained from numerical results compared to $2183 \mathrm{~W}$ obtained from analytical results.

Dielectric losses are validated using the capacitances calculated numerically with equation (24). A total of $166 \mathrm{~W}$ was obtained analytically, whereas dielectric losses using numerical calculation are $159 \mathrm{~W}$. Since the dielectric losses are mainly due to the parasitic capacitance between primary and secondary windings, the impact of the error on primary winding capacitance is negligible regarding losses.

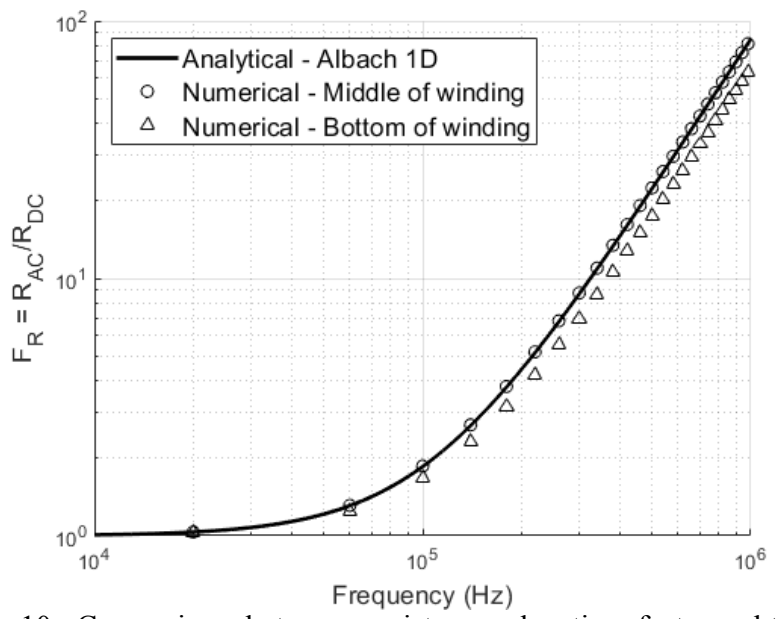

Fig. 10: Comparison between resistance elevation factors obtained analytically and numerically

3) Thermal Calculation: A 3D FEM thermal conduction simulation was built. The anisotropy of core and windings in terms of thermal conductivity was taken into account with local curvilinear coordinate systems. Homogeneous loss densities are applied to the magnetic core and to the windings. Each external surface of the transformer has a boundary condition applying the convection coefficients determined from empirical values.

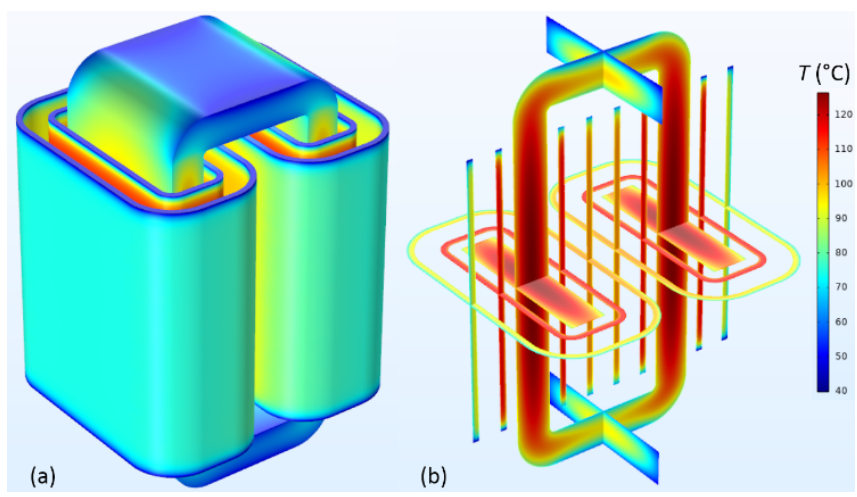

Fig. 11: Temperatures obtained via 3D FEM thermal simulation for the most compact design. (a) Surface temperature (b) Cross-sections temperature

This simulation was performed for the most compact design (Figure 11), but also for two designs with higher efficiency and volume (black circles from the Pareto front of Figure 7). The results are shown in Table 5. Taking into account that the ambient temperature is $40{ }^{\circ} \mathrm{C}$, it means the analytical model has an accuracy of about $10 \%$ for the temperature elevation, which is acceptable in the case of a thermal network approach. The accuracy of the analytical thermal model could be increased by adding more nodes to the thermal network, but it would result in a considerably higher calculation time, and the thermal calculation is already taking $70 \mathrm{~s}$ out of the total $72 \mathrm{~s}$. 
TABLE 5: MAXIMAL TEMPERATURES FOR THREE DESIGNS

\begin{tabular}{|c|c|c|c|c|c|c|c|}
\hline \multirow{2}{*}{$\begin{array}{c}\text { Volume } \\
\left(\mathrm{dm}^{3}\right)\end{array}$} & \multirow{2}{*}{ Efficiency } & \multicolumn{2}{|c|}{ Core } & \multicolumn{2}{c|}{ Primary Wind. } & \multicolumn{2}{|c|}{ Secondary Wind. } \\
\cline { 3 - 8 } & & Model & FEM & Model & FEM & Model & FEM \\
\hline 25.6 & $99.60 \%$ & $118{ }^{\circ} \mathrm{C}$ & $126^{\circ} \mathrm{C}$ & $116^{\circ} \mathrm{C}$ & $117^{\circ} \mathrm{C}$ & $112^{\circ} \mathrm{C}$ & $104^{\circ} \mathrm{C}$ \\
\hline 50.0 & $99.76 \%$ & $82^{\circ} \mathrm{C}$ & $88^{\circ} \mathrm{C}$ & $76^{\circ} \mathrm{C}$ & $76^{\circ} \mathrm{C}$ & $75^{\circ} \mathrm{C}$ & $71^{\circ} \mathrm{C}$ \\
\hline 100.0 & $99.80 \%$ & $69^{\circ} \mathrm{C}$ & $72^{\circ} \mathrm{C}$ & $54^{\circ} \mathrm{C}$ & $55^{\circ} \mathrm{C}$ & $56^{\circ} \mathrm{C}$ & $54^{\circ} \mathrm{C}$ \\
\hline
\end{tabular}

A summary of the comparison of transformer main characteristics between values obtained analytically and numerically is available in Table 4. The deviation is acceptable for each parameter and therefore the transformer design is validated.

\section{TeChNOlogical Choices AND Frequency ANALYSIS}

\section{A. Technological Choices Comparison}

Considering the application treated in paragraph III.A, other technological choices have been considered and studied following the same methodology. The goal here is to cover a lot of possible technological combinations to find the optimal one for this application. From the Table 1, all combinations of Core-Type Litz designs have been performed with either ferrite 3C93 or nanocrystalline Vitroperm 500F cut cores, which can both perform correctly up to $120^{\circ} \mathrm{C}$. It allowed to compare the insulation and cooling technologies between them. From this study, it appears that some combinations of insulation and cooling technologies were very promising, whereas others were performing really badly in terms of both compactness and efficiency. Therefore, to reduce the number of cases to study, only the best insulation and cooling technologies were retained, that is resin insulation with air-forced or cold plate cooling and oil insulation with forced oil cooling. With this reduced set of insulation and cooling technologies, the shell-type and foil technologies were studied. In addition, some designs with high-performance $\mathrm{SiFe}$ steel sheets (JNEX900, 6.5\% Si, $0.10 \mathrm{~mm}$ ) were also considered for reference.

In total, 39 technological choices combinations were studied, and 8 of them were excluded due to temperature restrictions. Moreover, all designs with $\mathrm{SiFe}$ steel sheets had an efficiency below $99 \%$ and would therefore not respect the criteria defined for this application. The Table 6 shows the volume, weight and efficiency of the most compact designs for each technological choices combination. Results are sorted from most compact to less compact ones. The best design in terms of compactness is the Core-Type, Litz, nanocrystalline with oil for insulation and cooling, which was the one presented in details and numerically validated in section III.
TABle 6: Designs for Various Technological Choices

\begin{tabular}{|c|c|c|c|c|c|c|c|c|}
\hline $\mathrm{N}^{\circ}$ & Geo. & Cond. & Mag. & Ins. & Cool. & \begin{tabular}{|c|}
$\begin{array}{c}\text { Volume } \\
\left(\mathrm{dm}^{3}\right)\end{array}$ \\
\end{tabular} & $\begin{array}{c}\text { Weight } \\
(\mathrm{kg})\end{array}$ & $\begin{array}{l}\text { Eff. } \\
(\%)\end{array}$ \\
\hline 1 & $\mathrm{CT}$ & Litz & Nano. & Oil & OF & 25.7 & 45.3 & 99.60 \\
\hline 2 & ST & Litz & Nano. & Oil & OF & 27.2 & 57.6 & 99.60 \\
\hline 3 & ST & Litz & $3 \mathrm{C} 93$ & Oil & OF & 30.5 & 74.7 & 99.70 \\
\hline 4 & $\mathrm{CT}$ & Litz & 3C93 & Oil & OF & 30.8 & 59.2 & 99.66 \\
\hline 5 & $\mathrm{CT}$ & Foil & Nano. & Oil & OF & 35.2 & 65.4 & 99.31 \\
\hline 6 & $\mathrm{CT}$ & Foil & 3C93 & Oil & OF & 42.1 & 97.0 & 99.57 \\
\hline 7 & ST & Foil & Nano. & Oil & OF & 50.9 & 327 & 99.05 \\
\hline 8 & $\mathrm{CT}$ & Litz & $\mathrm{SiFe}$ & Oil & OF & 55.0 & 118 & 98.99 \\
\hline 9 & ST & Foil & 3C93 & Oil & OF & 73.3 & 435 & 99.21 \\
\hline 10 & ST & Litz & $\mathrm{SiFe}$ & Oil & OF & 74.3 & 153 & 98.55 \\
\hline 11 & $\mathrm{CT}$ & Litz & 3C93 & Resin & $\mathrm{AF}$ & 85.4 & 184 & 99.74 \\
\hline 12 & ST & Litz & 3C93 & Resin & $\mathrm{AF}$ & 98.8 & 252 & 99.75 \\
\hline 13 & $\mathrm{CT}$ & Litz & 3C93 & Resin & $\mathrm{CP}$ & 108 & 205 & 99.79 \\
\hline 14 & CT & Foil & $\mathrm{SiFe}$ & Oil & $\mathrm{OF}$ & 115 & 282 & 98.02 \\
\hline 15 & CT & Litz & Nano. & Resin & $\mathrm{AF}$ & 115 & 227 & 99.55 \\
\hline 16 & ST & Litz & Nano. & Resin & $\mathrm{AF}$ & 116 & 340 & 99.71 \\
\hline 17 & ST & Litz & 3C93 & Resin & $\mathrm{CP}$ & 145 & 324 & 99.82 \\
\hline 18 & $\mathrm{CT}$ & Litz & Nano. & Resin & $\mathrm{CP}$ & 158 & 325 & 99.70 \\
\hline 19 & ST & Litz & Nano. & Resin & $\mathrm{CP}$ & 212 & 507 & 99.76 \\
\hline 20 & $\mathrm{CT}$ & Foil & 3C93 & Resin & $\mathrm{AF}$ & 229 & 556 & 99.36 \\
\hline 21 & $\mathrm{CT}$ & Foil & Nano. & Resin & $\mathrm{AF}$ & 244 & 541 & 99.14 \\
\hline 22 & $\mathrm{CT}$ & Litz & 3C93 & Air & $\mathrm{AF}$ & 249 & 130 & 99.69 \\
\hline 23 & $\mathrm{CT}$ & & 3C93 & Resin & AN & 265 & 586 & 99.44 \\
\hline 24 & $\mathrm{CT}$ & Litz & Nano. & Air & $\mathrm{AF}$ & 271 & 144 & 99.66 \\
\hline 25 & $\mathrm{CT}$ & Litz & 3C93 & AR & $\mathrm{AF}$ & 280 & 139 & 99.72 \\
\hline 26 & $\mathrm{CT}$ & Litz & Nano. & $\mathrm{AR}$ & $\mathrm{AF}$ & 303 & 136 & 99.62 \\
\hline 27 & $\mathrm{CT}$ & Foil & 3C93 & Resin & $\mathrm{CP}$ & 323 & 661 & 99.19 \\
\hline 28 & $\mathrm{CT}$ & Litz & $3 \mathrm{C} 93$ & Air & $\mathrm{AN}$ & 326 & 255 & 99.79 \\
\hline 29 & $\mathrm{CT}$ & Litz & $3 \mathrm{C} 93$ & $\mathrm{AR}$ & $\mathrm{AN}$ & 355 & 242 & 99.79 \\
\hline 30 & $\mathrm{CT}$ & Litz & Nano. & Air & $\mathrm{AN}$ & 398 & 380 & 99.75 \\
\hline 31 & $\mathrm{CT}$ & Litz & Nano. & AR & $\mathrm{AN}$ & 425 & 352 & 99.73 \\
\hline 32 & $\mathrm{CT}$ & Litz & Nano. & Resin & $\mathrm{AN}$ & \multicolumn{3}{|c|}{ No design $<120^{\circ} \mathrm{C}$} \\
\hline 33 & $\mathrm{CT}$ & Litz & $\mathrm{SiFe}$ & Resin & $\mathrm{AF}$ & \multicolumn{3}{|c|}{ No design $<120^{\circ} \mathrm{C}$} \\
\hline 34 & $\mathrm{CT}$ & Foil & Nano. & Resin & $\mathrm{CP}$ & \multicolumn{3}{|c|}{ No design $<120^{\circ} \mathrm{C}$} \\
\hline 35 & ST & Foil & Nano. & Resin & $\mathrm{AF}$ & \multicolumn{3}{|c|}{ No design $<120^{\circ} \mathrm{C}$} \\
\hline 36 & ST & Foil & Nano. & Resin & $\mathrm{CP}$ & \multicolumn{3}{|c|}{ No design $<120^{\circ} \mathrm{C}$} \\
\hline 37 & ST & Foil & 3C93 & Resin & $\mathrm{AF}$ & \multicolumn{3}{|c|}{ No design $<120^{\circ} \mathrm{C}$} \\
\hline 38 & ST & Foil & $3 \mathrm{C} 93$ & Resin & $\mathrm{CP}$ & \multicolumn{3}{|c|}{ No design $<120^{\circ} \mathrm{C}$} \\
\hline 39 & ST & Foil & $\mathrm{SiFe}$ & Oil & OF & \multicolumn{3}{|c|}{ No design $<120^{\circ} \mathrm{C}$} \\
\hline
\end{tabular}

CT: Core-Type, ST: Shell-Type, AR: Air-Resin, OF: Oil Forced, AF: Air Forced, AN: Air Natural, CP: Cold Plates.

Most compact designs have all in common to use oil insulation and cooling, which seems to be a prerequisite for this application if compactness is neededthe most -constrained.property. Inside those solutions, Core-Type geometries seem to be slightly better than Shell-Type ones, Litz better than foil and nanocrystalline better than ferrite 3C93. As discussed before, SiFe solutions have_a too low efficiency for this application, even though they can achieve quite good compactness.

However, oil solution may not be the best ones overall because of additional disadvantages like oil tank, pump management, complex manufacturing processes to ensure dry oil, maintenance, etc. Therefore another solution using dry-type insulation might be considered for these reasons even though the transformer designs give higher volumes. In this case, resin insulation with air forced cooling is the best combination, and nanocrystalline solutions fall behind ferrite ones. Core-Type is still better than Shell-Type with a more noticeable difference, and Litz better than foil. Cooling using cold plates is behind 
air forced cooling, mainly because of the size of the cold plates in the total volume. Moreover, natural air cooling does not seem to have enough cooling power for this application. In the end, it shows that cooling is a crucial aspect of MFT design and therefore most designs, at least for this application, are limited by thermal reasons.

In this technological choices comparison, cost was not included. Material costs can be easily integrated into such a design tool, because the weight of each material is known. It would only require to make hypotheses on the costs per kilogram for each material (core, windings, solid and/or liquid insulation). Because such hypotheses are not constant in time, but debatablefollowing the market prices, we chose not to include it in this publication. Moreover, these costs would not include processing costs, which are harder to evaluate.

It should be noted that all these conclusions on technological choices are closely linked to the case study. However, the tendency should remain the same for applications within the same range of rated power, insulation and frequency. For applications with significant differences in the specifications, this study can be performed again with the new inputs in a limited time thanks to the automated design tool that has been developed.

\section{B. Optimal Operating Frequency}

For this application, an analysis of the dependence of transformer performances on operating frequencies has been performed. This analysis constitutes a modification of the specifications and is only for investigation purposes. Moreover, the optimal operating frequency will be given regarding only the transformer properties, and therefore is probably not the optimal frequency for the complete converter. A large frequency range from $1 \mathrm{kHz}$ to $100 \mathrm{kHz}$ is taken, and only the case Core-Type, Litz, nanocrystalline core with oil for insulation and cooling is considered. It is not said that this specific structure is the best one over this whole frequency range, and it is probably not the case. However, this analysis can still give good insights on the optimal operating frequency, especially in the case of small frequency variations around $20 \mathrm{kHz}$. The calculation was performed for 200 frequency points, and for each frequency, a total of 16.2 million designs were calculated to determine the optimal design for this frequency. The results in terms of volume and losses are presented in Figure 12. The first thing that can be seen on these figures, particularly on losses, is that there are some discontinuities in the curves. This is explained by the discretization of the design space: each degree of freedom can only take a value amongst predefined ones. To enhance the continuity of the curves, either a finer discretization or a more complex optimization algorithm must be used. However, these curves are accurate enough to draw some conclusions. It appears that for this structure, the optimal operating frequency in terms of compactness is around $30-40 \mathrm{kHz}$.
Above this frequency, both volume and losses start to increase, which means there is no need to increase the frequency to such levels with such technologies.
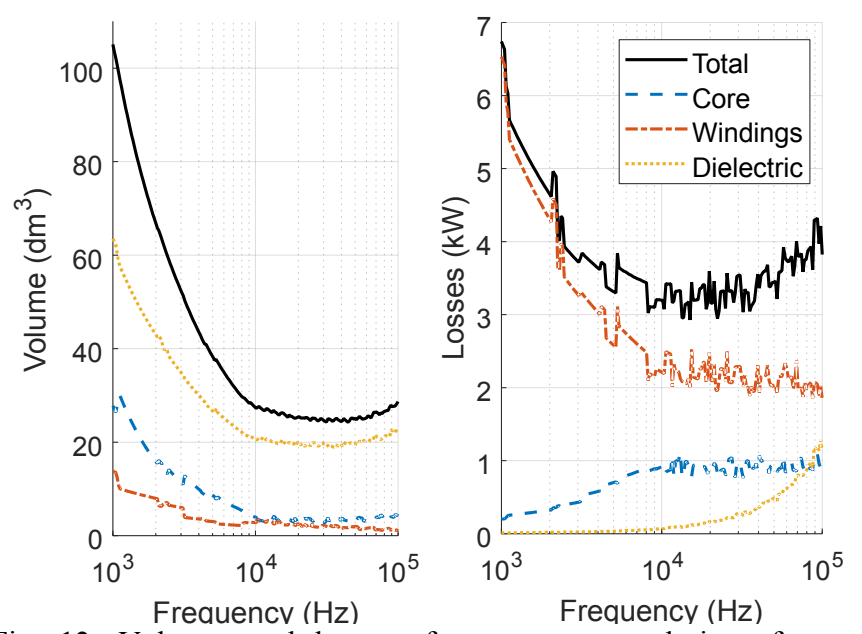

Fig. 12: Volumes and losses of most compact designs for each frequency, with Core-Type geometry, Nanocrystalline core, Litz cables, Oil insulation and cooling

This behavior is similar to the results found in [33-34] and reinforces the existence of an optimal operating frequency for MFT. Moreover, the gain in compactness is much more important below $10 \mathrm{kHz}$ than above. Because of the switching losses in converters, it may be better to work around $10 \mathrm{kHz}$ instead of $20-40 \mathrm{kHz}$. Regarding losses, there is a significant decrease below $10 \mathrm{kHz}$ for both core losses and windings losses, the dielectric losses being negligible for these low frequencies. However, above $10 \mathrm{kHz}$, total losses reach a plateau up to $40 \mathrm{kHz}$, from which losses start to increase mainly due to dielectric losses that increase rapidly for high frequencies.

\section{CONCLUSION}

A complete medium frequency transformer design methodology has been established to define the necessary steps in MFT design, aiming to efficiently cover a wide array of technologies. Based on this methodology, automated design tools have been developed to cover the most common technological choices of MFT and to be compatible with any voltage and current waveforms, and so any converter topology, which is an evolution from design methodologies of the literature. This implementation was performed using models adapted to the MFT requirements, coming either from literature or from our own developed models [19]. A focus has been made on optimizing the computation time with regards to the required accuracy. This is especially true for the analytical thermal modelling because of its major importance in the MFT performances, which are most of the time thermally limited, and its usually high computation cost. The developed design tool allows to cover more than one million designs in about one minute with a satisfying accuracy, which is an improvement with regards to the state of the art. Moreover, our approach 
takes into consideration certain properties that are usually neglected or not considered such as parasitic capacitances and dielectric losses.

This methodology was applied to a realistic application corresponding to a DC-DC converter in the range of some megawatts, for offshore windfarm application. An optimal design was found thanks to the analytical design tool, and was then verified by various FEM simulations. The comparative results between analytical and numerical models are in good agreement, and therefore constitute a first validation of the models used. However, some improvements may be achieved for parasitic capacitances calculations to take into account the presence of the magnetic core. Following this study on a specific MFT structure, a more global study was performed for a lot of possible technological choices combinations. It allowed to show the potential of a multistructure design methodology, and the important variations between structures that can lead to huge differences in terms of performances. Therefore, in order to optimally design a MFT, such approach is mandatory. Finally, a study regarding performances of a specific structure over a wide frequency range was performed. It showed that an optimal frequency does exist, above which there is no point in increasing the frequency anymore to reduce the MFT size.

As further work, additional structures variations may be integrated into the design tool with corresponding models to allow for an even better multi-structure tool. Also, the use of an optimization algorithm instead of a complete discretization of the design space might be an improvement in some cases, for example when the MFT performances to be optimized are clearly defined or when trying to find an optimal operating frequency. However, the discretization of the design remains a good first approach, as it draws a map of the possible designs and compromises. Finally, a comparison of calculation results following this methodology with experimental measurements on a manufactured MFT would be very useful to further validate the models. While manufacturing a prototype only to validate the models would be too expensive, a prototype manufactured for a more global project might be used in the future to validate the models.

\section{APPENDIX A}

The Table 7 below lists the correlations between dimensionless number, typically involving Nusselt number $\mathrm{Nu}$, Prandtl number $\mathrm{Pr}$ and either Rayleigh number $R a$ for natural convection or Reynolds number $R e$ for forced convection. Determining Nusselt number is equivalent to determine the convection coefficient. It also defines how the characteristic length (used in the expression of some dimensionless numbers) can be calculated for each type of face, and the correlations being used for both natural convection and forced convection.
For parallel plates, the channel width considered in the design tool is $10 \mathrm{~mm}$.

TABLE 7: DIMENSIONLESS NUMBERS CORRELATIONS

\begin{tabular}{|c|c|c|c|}
\hline & Face type & $\begin{array}{l}\text { Characteristic } \\
\text { Length }\end{array}$ & Correlation \\
\hline \multirow{4}{*}{ 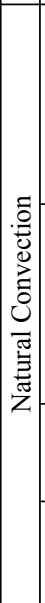 } & $\begin{array}{c}\text { Vertical Hot } \\
\text { Plate }\end{array}$ & Plate height & $\begin{aligned} N u=0.59 R a^{1 / 4} & \text { if } \\
N u=0.13 R a^{1 / 3} & \text { if } \\
R a<10^{9} & \\
R a>10^{9} & \end{aligned}$ \\
\hline & $\begin{array}{l}\text { Horizontal } \\
\text { Hot Plate - } \\
\quad \text { Top }\end{array}$ & $\begin{array}{c}\text { Ratio plate area } \\
\text { to plate } \\
\text { perimeter }\end{array}$ & $\begin{array}{cc}N u=0.54 R a^{1 / 4} & \text { if } \\
N u=0.15 R a^{1 / 3} & \text { if } \\
R a<10^{7} & \\
R a>10^{7} & \end{array}$ \\
\hline & $\begin{array}{l}\text { Horizontal } \\
\text { Hot Plate - } \\
\text { Bottom } \\
\end{array}$ & \begin{tabular}{|c|}
$\begin{array}{c}\text { Ratio plate area } \\
\text { to plate } \\
\text { perimeter }\end{array}$ \\
\end{tabular} & $N u=0.27 R a^{1 / 4}$ \\
\hline & \begin{tabular}{|c|} 
Vertical \\
Parallel Plates
\end{tabular} & $\begin{array}{c}\text { Distance } \\
\text { between plates }\end{array}$ & $N u=\left(\frac{576}{R a^{2}}+\frac{2.873}{R a^{0.5}}\right)^{-0.5}$ \\
\hline \multirow{4}{*}{ 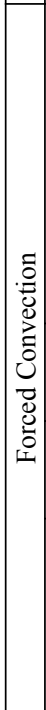 } & $\begin{array}{c}\text { Plate Parallel } \\
\text { to Flow }\end{array}$ & Plate length & $\begin{aligned} & \frac{N u}{2}=\left.\left.\frac{0.3387 \operatorname{Pr}^{1 / 3} \operatorname{Re}^{1 / 2}}{(1+(0.0468 /} / \operatorname{Pr}\right)^{2 / 3}\right)^{1 / 4} \\
& \text { if } \quad \operatorname{Re}<5.10^{5} \\
& \frac{N u}{2}= \operatorname{Pr}^{1 / 3}\left(0.037 \operatorname{Re}^{4 / 5}-871\right. \\
& \text { if } \quad \operatorname{Re}>5.10^{5}\end{aligned}$ \\
\hline & \begin{tabular}{|c|} 
Plate Face to \\
Flow
\end{tabular} & $\begin{array}{l}\text { Half of plate } \\
\text { smallest } \\
\text { dimension } \\
\end{array}$ & $N u=0.564 \operatorname{Re}^{1 / 2} \operatorname{Pr}^{1 / 3}$ \\
\hline & \begin{tabular}{|} 
Plate Back to \\
Flow
\end{tabular} & $\begin{array}{l}\text { Half of plate } \\
\text { smallest } \\
\text { dimension } \\
\end{array}$ & $N u=0.27 \operatorname{Re}^{0.7} \operatorname{Pr}^{1 / 3}$ \\
\hline & $\begin{array}{c}\text { Parallel Plates } \\
\text { Duct }\end{array}$ & $\begin{array}{c}\text { Twice the } \\
\text { distance } \\
\text { between plates }\end{array}$ & $\begin{array}{cc}N u=7.54 & \text { if } \\
N u=0.023 \operatorname{Re}^{0.8} \operatorname{Pr}^{1 / 3} & \text { if } \\
R e<4.10^{3} & \\
R e>4.10^{3} & \end{array}$ \\
\hline
\end{tabular}

\section{ACKNOWLEDGMENT}

This work was supported by a grant overseen by the French National Research Agency (ANR) as part of the "Investissements d'Avenir" Program (ANE-ITE-00201).

\section{REFERENCES}

[1] J. W. Kolar and G. Ortiz., "Solid-state-transformers: key components of future traction and smart grid systems," in Proceedings of the International Power Electronics ConferenceECCE Asia (IPEC 2014), 2014.

[2] A. Prasai, J.-S. Yim, D. Divan, et al., "A New Architecture for Offshore Wind Farms," IEEE Transactions on Power Electronics, vol. 23, pp. 1198-1204, May 2008.

[3] T. Lagier, P. Ladoux, "A comparison of insulated DC-DC converters for HVDC off-shore wind farms," in International Conference on Clean Electrical Power (ICCEP), 2015.

[4] J. E. Huber, J. W. Kolar, "Volume/weight/cost comparison of a 1MVA $10 \mathrm{kV} / 400 \mathrm{~V}$ solid-state against a conventional lowfrequency distribution transformer," in IEEE Energy Conversion Congress and Exposition (ECCE), 2014. 
[5] A. Garcia-Bediaga, I. Villar, A. Rujas, et al., "Multiobjective Optimization of Medium-Frequency Transformers for Isolated Soft-Switching Converters using a Genetic Algorithm," IEEE Transactions on Power Electronics, vol. 32, pp. 2995-3006, 2016.

[6] M. A. Bahmani, T. Thiringer, M. Kharezy, "Optimization and experimental validation of medium-frequency high power transformers in solid-state transformer applications," in IEEE Applied Power Electronics Conference and Exposition (APEC), 2016.

[7] M. Mogorovic, D. Dujic, " $100 \mathrm{~kW}, 10 \mathrm{kHz}$ Medium Frequency Transformer Design Optimization and Experimental Verification," IEEE Transactions on Power Electronics, 2018.

[8] M. Leibl, G. Ortiz, J. W. Kolar, "Design and Experimental Analysis of a Medium-Frequency Transformer for Solid-State Transformer Applications," IEEE Journal of Emerging and Selected Topics in Power Electronics, vol. 5, pp. 110-123, March 2017.

[9] P. Shuai, J. Biela, "Design and optimization of medium frequency, medium voltage transformers," in 15th European Conference on Power Electronics and Applications (EPE), 2013.

[10] P. Huang, C. Mao, D. Wang, et al., "Optimal Design and Implementation of High-voltage High-power Silicon Steel Core Medium Frequency Transformer," IEEE Transactions on Industrial Electronics, 2017.

[11] O. Aldosari, L. A. G. Rodriguez, J. C. Balda, et al., "Design Trade-Offs for Medium- and High-Frequency Transformers for Isolated Power Converters in Distribution System Applications," in 9th IEEE International Symposium on Power Electronics for Distributed Generation Systems (PEDG), 2018.

[12] S. Vaisambhayana, C. Dincan, C. Shuyu, et al., "State of art survey for design of medium frequency high power transformer," in Asian Conference on Energy, Power and Transportation Electrification (ACEPT), 2016.

[13] T. Kauder, K. Hameyer, "Performance Factor Comparison of nanocrystalline, amorphous and crystalline soft-magnetic Materials for medium-frequency Applications," IEEE Transactions on Magnetics, 2017.

[14] S. Zhao, Q. Li, F. C. Lee, "High frequency transformer design for modular power conversion from medium voltage $\mathrm{AC}$ to 400V DC," in IEEE Applied Power Electronics Conference and Exposition (APEC), 2017.

[15] M. Stojadinović, J. Biela, "Modelling and Design of a Medium Frequency Transformer for High Power DC-DC Converters," in International Power Electronics Conference (IPEC-Niigata 2018 -ECCE Asia), 2018.

[16] M. Jafari, Z. Malekjamshidi, M. R. Islam, et al., "Modeling of magnetic flux in multi-winding toroidal core high frequency transformers using 3D reluctance network model," in IEEE 11th International Conference on Power Electronics and Drive Systems (PEDS), 2015.

[17] S. Baek, S. Bhattacharya, "Analytical Modeling and Implementation of a Coaxially Wound Transformer with Integrated Filter Inductance for Isolated Soft-Switching DC-DC Converters," IEEE Transactions on Industrial Electronics, 2017.

[18] G. Ortiz, J. Biela, D. Bortis, et al., "1 Megawatt, $20 \mathrm{kHz}$, isolated, bidirectional $12 \mathrm{kV}$ to $1.2 \mathrm{kV}$ DC-DC converter for renewable energy applications," in 2010 International Power Electronics Conference (IPEC), 2010.

[19] A. Fouineau, M.-A. Raulet, B. Lefebvre, et al., "Semi-Analytical Methods for Calculation of Leakage Inductance and FrequencyDependent Resistance of Windings in Transformers," IEEE Transactions on Magnetics, vol. 54, pp. 1-10, Oct 2018.

[20] M. Mogorovic, D. Dujic, "Medium Frequency Transformer Leakage Inductance Modeling and Experimental Verification," in IEEE Energy Conversion Congress and Exposition (ECCE), 2017.

[21] J. Biela, J. W. Kolar, "Using Transformer Parasitics for Resonant Converters - Review of the Calculation of the Stray Capacitance of Transformers," IEEE Transactions on Industry Applications, vol. 44, pp. 223-233, Jan 2008.

[22] C. P. Steinmetz, "On the law of hysteresis," Transactions of the American Institute of Electrical Engineers, vol. 9, pp. 1-64, 1892.

[23] I. Villar, A. Rufer, U. Viscarret, et al., "Analysis of empirical core loss evaluation methods for non-sinusoidally fed medium frequency power transformers," in IEEE International Symposium on Industrial Electronics (ISIE), 2008.

[24] K. Venkatachalam, C. R. Sullivan, T. Abdallah, et al., "Accurate prediction of ferrite core loss with nonsinusoidal waveforms using only Steinmetz parameters," in IEEE Workshop on Computers in Power Electronics, 2002.

[25] M. Albach, "Two-dimensional calculation of winding losses in transformers," in IEEE 31st Annual Power Electronics Specialists Conference, 2000.

[26] P. L. Dowell, "Effects of eddy currents in transformer windings," Proceedings of the Institution of Electrical Engineers, vol. 113, pp. 1387-1394, August 1966.

[27] D. Gerling, G. Dajaku, "Novel lumped-parameter thermal model for electrical systems," in European Conference on Power Electronics and Applications (EPE), 2005.

[28] P. A. Kyaw, J. Qiu, C. R. Sullivan, "Analytical Thermal Model for Inductor and Transformer Windings and Litz Wire," in IEEE 19th Workshop on Control and Modeling for Power Electronics (COMPEL), 2018.

[29] W. M. Rohsenow, J. P. Hartnett, Y. I. Cho, et al., "Handbook of heat transfer," vol. 3, McGraw-Hill New York, 1998.

[30] M. Favre-Marinet, S. Tardu, "Convective Heat Transfer: Solved Problems," John Wiley \& Sons, 2013.

[31] M. Mogorovic, D. Dujic, "Thermal Modeling and Experimental Verification of an Air Cooled Medium Frequency Transformer," in 19th European Conference on Power Electronics and Applications (EPE'17 ECCE Europe), 2017.

[32] J. Maneiro, R. Ryndzionek, T. Lagier, et al., "Design of a SiC based triple active bridge ceil for a multi-megawatt DC-DC converter," in 19th European Conference on Power Electronics and Applications (EPE'17 ECCE Europe), 2017.

[33] U. Drofenik, "A $150 \mathrm{~kW}$ Medium Frequency Transformer Optimized for Maximum Power Density," in 7th International Conference on Integrated Power Electronics Systems (CIPS), 2012.

[34] M. Mogorovic, D. Dujic, "Sensitivity Analysis of Medium Frequency Transformer Designs for Solid State Transformers," IEEE Transactions on Power Electronics, 2018. 